\title{
Re-Mar: Repository of Marine Learning Objects
}

\author{
André Behr ${ }^{1}$, José Cascalho ${ }^{1,2}$, Hélia Guerra ${ }^{1,3}$, Ana Costa ${ }^{1,4}$, \\ Manuela Parente $^{1,4}$, Andrea Botelho ${ }^{1,4}$, Rosa Vicari ${ }^{5}$, Armando Mendes ${ }^{1,2}$ \\ ${ }^{1}$ FCT, Azores University, Ponta Delgada, Portugal \\ ${ }^{2}$ GRIA and LIACC, Portugal \\ ${ }^{3}$ NIDeS and Centro Algoritmi, Portugal \\ ${ }^{4} \mathrm{CIBIO}$ and InBIO, Portugal \\ ${ }^{5}$ Federal University of Rio Grande do Sul, Porto Alegre, Brazil \\ \{andre.r.behr, jose.m.cascalho, armando.b.mendes\}@uac.pt
}

\begin{abstract}
Current literature shows the lack of learning object repositories exclusively related to environmental education and that there is no predominant software. This paper presents Re-Mar, a marine learning object repository based on open source software. Re-Mar is a part of an effort to promote ocean literacy through educational content for students and teachers. The repository is supported by computational technologies to catalog and organize learning objects to retrieve and reuse. Our prototype shows that is possible to store, catalog, retrieve, and link learning objects to support environmental education and coping with learning objects lifecycle. This is the first step to future aggregation of linked data, ontologies, and artificial intelligence aspects.
\end{abstract}

\section{Introduction}

The continuous monitoring of natural resources and the management of their measures are extremely important for environment preservation so that sustainable exploration can be carried out [Cairns 2002]. Allied to this, the awareness of humanity must be grounded so that greater damage to nature could be avoided, such as species extinction, too much pollution, and global warming. Teachers are key players in engaging students to maintain the environment. They can integrate care to natural resources in their lessons [Ramadhan et al. 2019].

Digital technologies can help to involve students in environment preservation through learning practices, but effective environmental teaching will just be reached with engagement and familiarisation with the current digital technologies [Buchanan et al. 2018]. One of these digital technologies is Learning Objects (LOs) that play an important role in general education and can be applied to environmental education.

Learning Objects can be employed in different contexts and mixed to generate a brand new LO. They are usually stored and cataloged by metadata in Learning Object Repositories (LORs) to improve the use and reuse [Vicari et al. 2010].

Repositories of learning objects are also considered the first step to build an ecosystem for Digital Education with Artificial Intelligence techniques. Open architec- 
tures, metadata standards, communication protocols, and policies are the main challenges to achieve this kind of proposed ecosystem [Coelho and Primo 2017].

In this way, the LO lifecycle must be considered since a unit to elementary analysis of services that will be implemented to LOs. [Gluz and Vicari 2014] depict three main phases: (i) LO authoring that leads with content creation/edition, encapsulation/packaging, metadata creation/edition; (ii) LO usage that handles search and find the appropriate object, availability and support for use (students), monitoring of use (teachers); and (iii) LO maintenance to support content/metadata management, version management, adaptations for multiple platforms, and adaptations for accessibility requirements.

This ongoing work will focus on environmental education, especially related to the oceans. We will present a learning object repository supported by computer technologies to foster ocean literacy that handles most aspects of the learning objects' lifecycle.

The text follows with a theoretical background about the main subjects addressed. Then, the SeaThings project and its relation with ocean literacy through learning objects with a metadata standard will be presented. Next, a section with the working progress on building a learning object repository called Re-Mar. In the end, the conclusion and future work will be discussed.

\section{Theoretical Background}

\subsection{SeaThings Project}

The improvement of ocean literacy is the main focus of the SeaThings project. To provide educational resources, a LOR will be applied to arouse curiosity and increase knowledge. These will allow the development of more informed, more responsible, and more participative citizens.

SeaThings project has three main areas. The educational sciences are a fundamental part of the project as it aims to produce and making available materials by teachers and students to use in classes related to ocean subjects. Natural sciences are the scientific area of the materials to be produced. The technology used in SeaThings is represented by the computer and information sciences, supporting the management of the online repository and artificial intelligent-based supportive tools.

Due to regional context aspects, it has the promotion of ocean literacy with an emphasis on Azorean marine environments and dissemination and propelling of scientific knowledge throughout teaching, instigating scientific curiosity among young people. It also reinforces the importance of marine environmental monitoring as a management measure, aimed at the sustainable exploitation of Atlantic marine resources.

The project has the goal to provide LOs for the different levels of education. The participation of the schools and science centers will contribute to populating the LOR. There are no restrictions to the level of the LOs produced, although the identification of the LO target is expected.

\subsection{Learning Objects and Sequencing}

There are several definitions for Learning Objects (LOs). One of them is proposed by the Learning Technology Standards Committee (LTSC) that states a LO as "any entity, digital or non-digital, that may be used for learning, education or training" and cites learning 
objectives, multimedia content, instructional content, instructional software and software tools, and people, organizations, or events referenced during technology supported learning as examples of LOs [LTSC 2002]. Willey classified this definition as "extraordinarily broad" and proposed "a learning object as any digital resource that can be reused to support learning”, rejecting non-digital and non-reusable resources [Wiley 2000].

Instruction based on the design of LOs can be supported by its scope and sequence recommendations. Also, LOs must be sequenced according to any dependencies discovered, such as complexity, difficulty, level, and type [Wiley 2000].

Learning Objects Repositories (LORs) aim to improve LO reuse, organizing several LOs. LOs are usually cataloged to enhance the LOR retrieving ability. In this way, several international standards have been created, tagging LO with metadata. As examples of widely used metadata standards, we can cite Dublin Core [DCMI 2020] that is a generic standard and IEEE-LOM [LTSC 2002] that is an educational-oriented standard.

The LO lifecycle phases are similar to other types of technological artifacts. But they have division levels at content and metadata. Also, there are concerns with interoperability in multiple digital platforms and accessibility aspects [Gluz and Vicari 2014].

\subsection{OBAA Metadata Standard}

The Agent-based Learning Objects (OBAA) [Vicari et al. 2010] proposes to extend the traditional IEEE-LOM. OBAA aggregates several new metadata to allow the representation of any LO, since simple texts such as lesson plans and even interactive games. This standard is also compatible with the Web, mobile devices, and digital television. OBAA has more than one hundred metadata divided into 11 categories: general, lifecycle, metametadata, technical, educational, rights, relation, annotation, classification, accessibility, and segment information table.

For educational aspects, it is possible to describe didactic strategies, interactions, perceptions, learning content types, among others. If necessary, accessibility aspects can be informed, as if the LO is oriented for blind users, a caption for the deaf, and other languages.

As in IEEE-LOM standard, it is also possible to make relationships between learning objects through metadata. In addition to catalog and entry metadata, the kind of relation can be specified, such as: is part of, has a part, is a version of, has a version, is a format of, has a format, references, is referenced by, is based on, is the basis for, requires, and is required by.

Adopting the OBAA metadata, it is expected to describe several types of learning objects. It will also help to support control and integration among LOs. Besides, OBAA provides compatibility to Agents and Ontologies technologies, been prepared for the current Web extension [Behr et al. 2012].

\section{Related Work}

SeaThings project [Mendes 2020] will firstly emphasize the selected main areas: Biodiversity, Ecology, Threats/Impacts, and Management. The focus will be given to the valuation and conservation of the ocean, and sustainable use by encouraging good practices in the marine environment. Learning objects related with biodiversity and ecology 
(e.g. intertidal, subtidal, deep-sea environments but also Marine Protected Areas - MPAs) are essential elements for understanding the functioning of marine ecosystems and will be part of the repository material supported by OBAA metadata.

We made an exploratory research looking for LORs that are related to the SeaThings context. First, two sources of indexing LORs were explored. Then, a literature review of the state-of-the-art was done.

To retrieve LORs, the Registry of Open Access Repository (ROAR) ${ }^{1}$ and the Directory of Open Access Repositories (Open DOAR) ${ }^{2}$. ROAR was filtered with "Learning and Teaching Objects" for "Repository Type" and retrieved 76 LORs. Open DOAR was filtered with "Learning Objects" for "Content Type" and "Science General" for "Subjects", retrieving 176 LORs.

Analyzing the resultant records, discarting the LORs that are offline and that are not related to SeaThings context, Table 1 presents the results. It is important to be said that several institutional repositories were retrieved, but they are only considered if they have a specific collection for learning objects.

Table 1. LORs characteristcs on the theme of SeaThings context

\begin{tabular}{|l|l|l|}
\hline LOR Name & Related Collections & Software \\
\hline Biblioteca Nacional de Maestros & Rural Education & Other \\
\hline $\begin{array}{l}\text { Universidad Politécnica } \\
\text { Salesiana Ecuador }\end{array}$ & Natural and Exact Sciences & DSpace \\
\hline Scout Archives & - & Other \\
\hline $\begin{array}{l}\text { Repositorio de Recursos } \\
\text { Educativos Abiertos de Ceibal }\end{array}$ & Life Sciences / Environment & Other \\
\hline openNCCC & Agriculture \& Natural Sciences & ISKME \\
\hline National Science Digital Library & $\begin{array}{l}\text { Agriculture / Geology / } \\
\text { Biology /Ecology }\end{array}$ & ISKME \\
\hline $\begin{array}{l}\text { EERL - Enviromental \& } \\
\text { Energy Resources Library }\end{array}$ & $\begin{array}{l}\text { Natural Resources Management / } \\
\text { Pollution Prevention / } \\
\text { Sustainability / ... }\end{array}$ & Other \\
\hline
\end{tabular}

In [Saltidou and Skoumios 2017] work, the Greek Digital Learning Object Repository was the subject of study. The authors evaluate if LOs engage the students in science practices. This LOR is intended for primary and secondary school. It has related collections as Geography-Geology, Biology, and Environmental Education/Education for Sustainable Development. As functionalities, it supports browsing, free text search, and faceted search, allowing users to narrow search results by applying multiple filters, such as learning resources type, educational context, etc. It is powered by DSpace software.

[Pounds and Bostock 2019] employed questionnaires to evaluate LOs related to aquaculture and fishery and their potential benefits. They cited in their work The European Commission's VOARAA ${ }^{3}$ and AquaCase $3.0^{4}$ projects that produced LOs related to

\footnotetext{
${ }^{1}$ http://roar.eprints.org/

${ }^{2}$ https://v2.sherpa.ac.uk/opendoar/

${ }^{3}$ https://cordis.europa.eu/project/rcn/204632/en

${ }^{4}$ http://www.aquacase.org/
} 


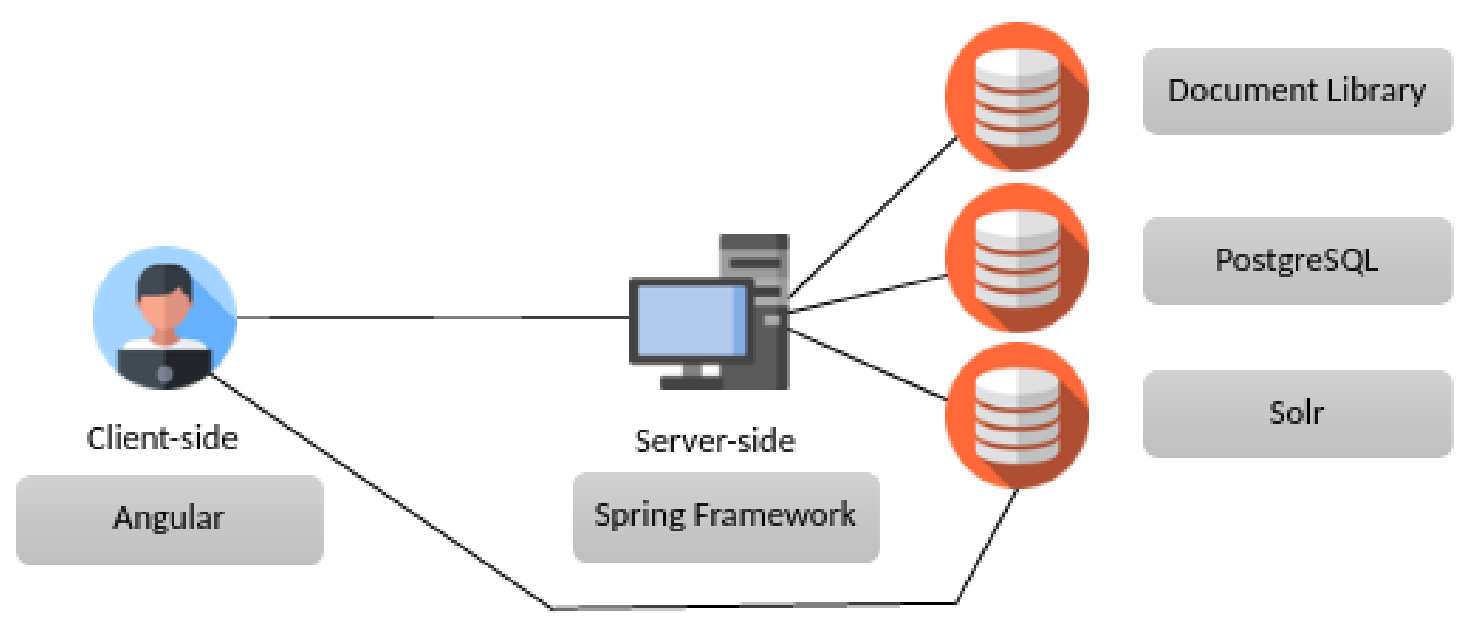

Figure 1. Client-server architecture and technologies

aquaculture and fisheries. VOARAA is based on a community-focused integrated service and a social portal that has different search options available, such as tag-based, textbased, navigational, social, and author. The AquaCase site provides supplemental material to classroom teaching and it is divided by categories, without searching functionality. Both sites do not indicate the software employed.

The current literature review shows the lack of works of LORs exclusively related to environmental education and that there is no predominant software for it. Also, many universities have institutional repositories registered at ROAR and Open DOAR typed as a Learning Object but are actually used more as a publication repository.

Our work differs from related works providing a solution that completely separates back-end and front-end to provide marine learning objects. Besides that, a relation-based control and integration are explored for sequencing LOs and pre-fill metadata.

\section{Re-Mar}

Re-Mar uses an open-source software for learning object repositories called Cognix, customizing its back-end ${ }^{5}$ and front-end ${ }^{6}$. It furnishes a graphical interface accessed by web browsers to submit and search learning objects.

\subsection{Architecture}

A client-server architecture supports Re-Mar. It is possible to exchange messages between the client-side and the server-side. All the messages are in JavaScript Object Notation (JSON) format.

The communication between client-server and used technologies are depicted in Figure 1. For the client-side, Angular is employed, a platform and framework that uses HTML, CSS, and JavaScript. On the server-side, the Spring Framework based on Java and REST API is used.

The application also manages three kinds of databases. The first one, a Linuxbased Document Library, stores all the files related to the LO. The second, a PostgreSQL

\footnotetext{
${ }^{5}$ https://github.com/arbehr/Cognix

${ }^{6}$ https://github.com/arbehr/cognix-front-temp
} 
database, manage users, documents, and files information data. The third, Solr (a NoSQL database) is responsible for the LOR search engine.

Registered users can submit LOs to Re-Mar. The authentication and authorization are managed by a JSON Web Token (JWT) at the server-side. After login in Re-Mar, users acquire a token that gives privileges in the repository. This token is also necessary to search and insert documents in Solr. Besides the login, the back-end has end-points to send emails and manage files, users, and metadata.

With the JWT approach, it is possible to uncouple PostgreSQL and Solr of the same server. Then, if necessary, the databases could be distributed in different machines. That could be important if the databases, in some time, need to have specific machine configuration/hardware.

Beyond a metadata core for searching, we added cores to store statistics metrics in Solr, such as downloads and access. User searching terms are also saved to further analytics.

\subsection{Submition Workflow}

The submission workflow at Re-Mar follows the ordered steps below:

1. User Login: After registered, the user can log in with an e-mail and password.

2. Selecting files and filling in the form: The user chooses the LO file and a thumbnail. Fill in and select form fields. It is possible to save the form and continue later, at anytime.

3. LO submission: After filling all the fields, the user can submit the LO for revision.

4. Technical revision: It is the first revision. It is verified if the files are working and if the form was filled correctly.

5. Pedagogical revision: The second revision. It is checked the educational and pedagogical characteristics of the LO.

6. LO publication: LO is available at Re-Mar and the authors are informed of the fact.

\subsection{Control and Integration}

Despite download and access statistics are widely used, they do not evidence the real reuse of LOs. To reduce this drawback, the use of relation metadata is embraced. LOs in Re-Mar will have version control and could have integration among them. That will be possible through the OBAA lifecycle and relation metadata.

Figure 2 shows its interface to the LO relationship. (1) It is asked if the current LO was based on a previous LO in the repository. (2) If yes, the user selects the kind of relation: have a part, version, reference, and requirement are handled. (3) More than one relationship can be added.

For example, suppose a book is submitted to Re-Mar. Sometime later, the authors also elaborate a class plan using this book, producing a new LO and would like to submit it in Re-Mar. After the relation metadata step, it is possible to retry all the related metadata from the used LO (for the book). So, the user can just edit the differences, like title and description, and maintain the other, like authors, knowledge areas, keywords, etc (for the class plan). 


\section{Relations between learning objects}

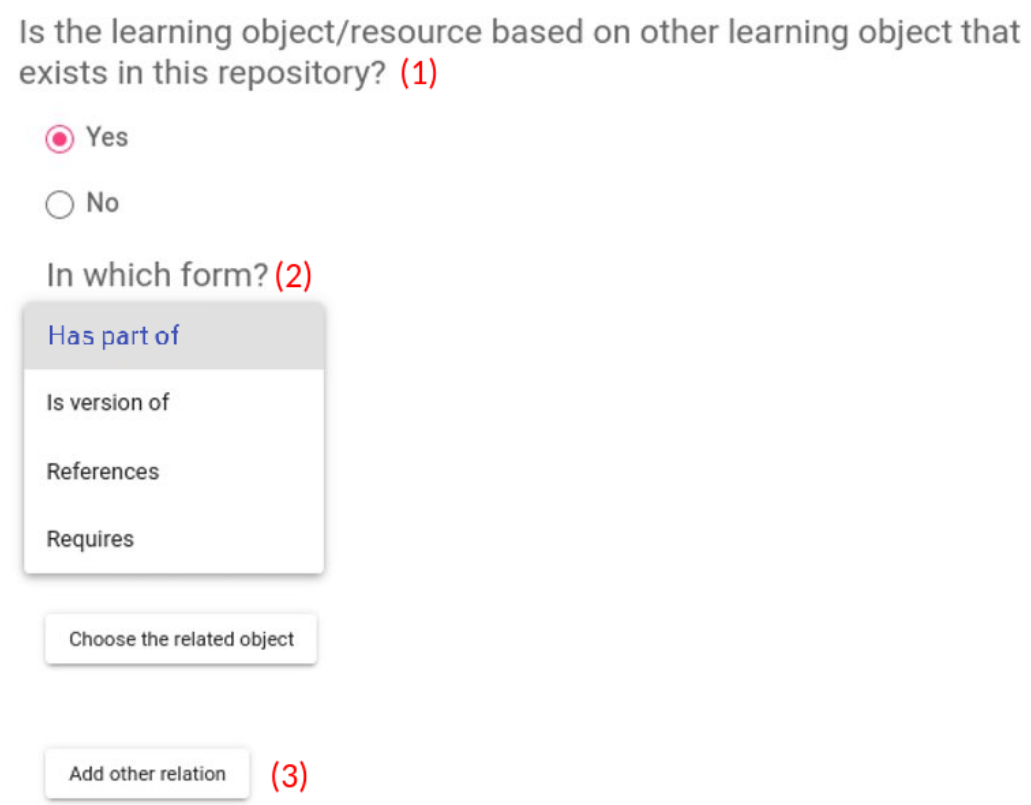

Figure 2. Interface to relation metadata

\subsection{Results}

The Re-Mar prototype is already available online at http://re-mar.uac.pt and has its home page depicted in Figure 3 but still very new and with only a few registered LOs. Its interface aims to be compatible with different devices and their capacities, like screen size and performance.

Figure 4 shows how the interface helps the user in filling up metadata with (1) keyword suggestions (pre-defined), but they can (2) add their own. The user can also import all the metadata and pre-fill the form with metadata retrieved from the related LO.

Several types of LOs can be submitted to the repository, such as videos, images, texts, audios, animations, simulations, and games. All of them must be share in an open license. The licenses provided are Creative Commons (CC) BY-SA and BY-NC-SA, both allowing reuse and free access.

Re-Mar provides communication protocols that are available to other repositories or applications to share its LOs. Current, Open Archives Initiative Protocol for Metadata Harvesting (OAI-PMH) and REST API are accessible at the server-side.

Figure 5 exemplifies a search in Re-Mar where the user does a query with the "natur" string. The repository looks for this string in title, keywords, and description metadata fields. Users can share the LOs in social media, such as Facebook, Twitter, and Linkedin. They also can "like" their favorite LOs. The "+ Information" link provides all the metadata and a download link to the LO.

Re-Mar employs a subset of OBAA metadata standard that contains the following main groups: General, LifeCycle, Meta-Metadata, Technical, Educational, Rights, and 


\section{$\stackrel{\text { Re-Mar }}{2}$}

Add your leaning objects, search, and share!

Search for learning objects

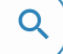

Learning object submission

Figure 3. Current responsive home page

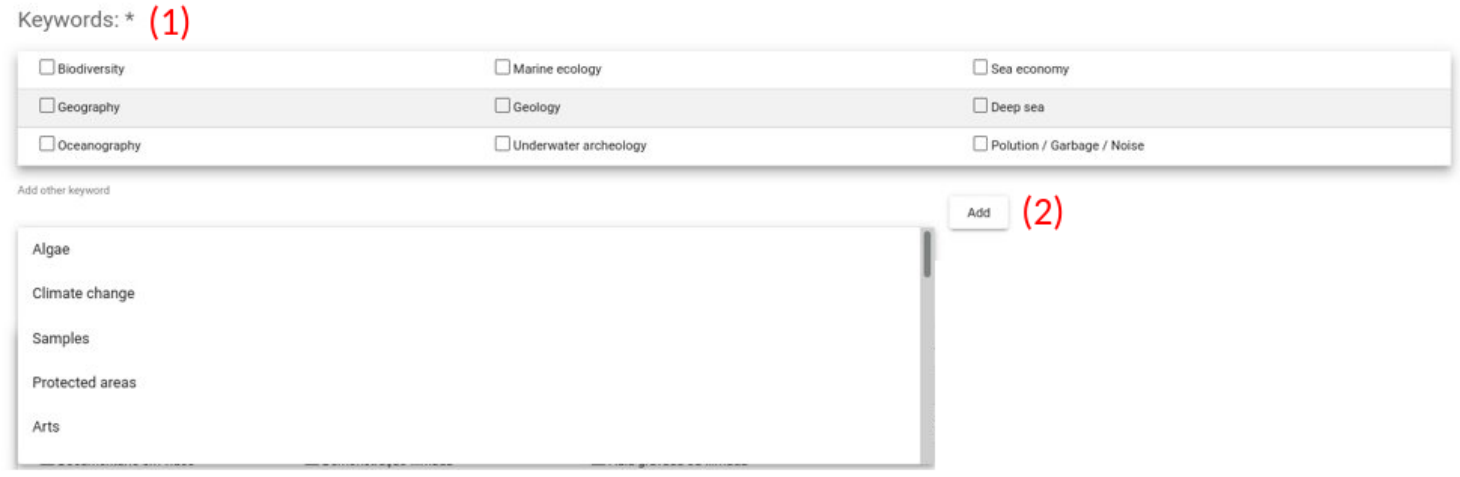

Figure 4. Keywords suggestions interface

Relation. The current subset can be reached at http://re-mar.uac.pt/metadata.html.

\section{Conclusion and Future Work}

LOs are important artifacts that can be used in learning and teaching. By grouping them in LORs, it is possible to enhance them with services for cataloging and searching through metadata to support LO reuse. However, nowadays, little LO material exists related to environmental education, especially in the oceans field. Almost all the LORs are multidisciplinary.

In this work, we presented a marine repository that completely separates back-end and front-end, providing easier maintenance. Besides that, the back-end could be used by other applications, such as a mobile app or a learning platform.

In addition to technical aspects, engaging the community to submit and use learning objects is capital for the LOR's success. SeaThings project aims to add LOs in Re-Mar by theme. It will invite teachers, students, and science promoters to test Re-Mar and its LOs to produce new LOs as part of classroom or workshop activities.

With a community of users, it will be possible to evaluate the Re-Mar interface and metadata. By usage tracking, metadata customization for each type of LO can be 


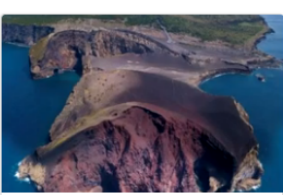

Conservação da natureza...

$0<$

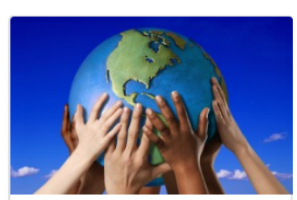

Recursos naturais renov..

$0<$

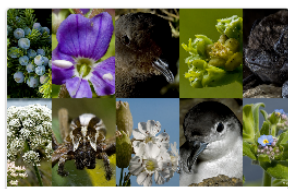

Açores - biodiversidade...

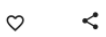

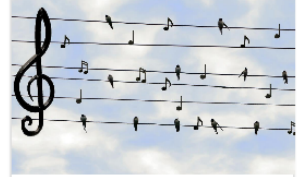

Música com ambiente: DJ...

$0<$

Figure 5. Search interface

done. It is also expected to provide a multilingual interface for geographically different users.

As the repository grows, new computational technologies can be incorporated in Re-Mar. For example, ontologies describe data and enhance the search for both humans and machines. Intelligent agents to do autonomous tasks, like metadata collection, reasoning, and recommendations.

\section{Acknowledgements}

This work is financed by the FEDER in $85 \%$ and by regional funds in $15 \%$, through the Operational Program Azores 2020, within the scope of the SEA-THINGS Learning Objects to Promote Ocean Literacy project ACORES-01-0145-FEDER-000110. This study was also financed in part by the Coordenação de Aperfeiçoamento de Pessoal de Nível Superior - Brasil (CAPES) - Finance Code 001. This work was partially funded by FEDER funds through the Operational Programme for Competitiveness Factors COMPETE and by National Funds through FCT - Foundation for Science and Technology under the UID/BIA/50027/2020 and POCI-01-0145-FEDER-006821, by funding the CIBIO/InBIO.

\section{References}

Behr, A., Primo, T., and Viccari, R. (2012). An ontology for the obaa metadata standard. In Brazilian Symposium on Computers in Education (Simpósio Brasileiro de Informática na Educação-SBIE), volume 23.

Buchanan, J., Pressick-Kilborn, K., and Maher, D. (2018). Promoting environmental education for primary school-aged students using digital technologies. Eurasia Journal of Mathematics, Science and Technology Education, 15(2):15.

Cairns, J. (2002). Environmental monitoring for the preservation of global biodiversity: the role in sustainable use of the planet. International Journal of Sustainable Development and World Ecology, 9(2):135-150.

Coelho, H. and Primo, T. T. (2017). Exploratory apprenticeship in the digital age with ai tools. Progress in Artificial Intelligence, 6(1):17-25. 
DCMI (2020). Dcmi metadata terms. Technical report. Accessed december 2020.

Gluz, J. C. and Vicari, R. M. (2014). Rumo a uma plataforma semântica de conteúdos educacionais digitais: o modelo ontológico. In Brazilian Symposium on Computers in Education (Simpósio Brasileiro de Informática na Educação-SBIE), volume 25, page 993.

LTSC (2002). Ieee standard for learning object metadata (draft). ieee standard 1484.12.1.

Mendes, A. (2020). Sea-things: A project to improve the ocean literacy in the azores. Arquipelago-Life and Marine Sciences, pages 51-52.

Pounds, A. and Bostock, J. (2019). Open educational resources (oer) in higher education courses in aquaculture and fisheries: opportunities, barriers, and future perspectives. Aquaculture International, 27(3):695-710.

Ramadhan, S., Sukma, E., and Indriyani, V. (2019). Environmental education and disaster mitigation through language learning. In IOP Conference Series: Earth and Environmental Science, volume 314, page 012054. IOP Publishing.

Saltidou, E. and Skoumios, M. (2017). Science practices in the science learning objects of the greek digital learning object repository. Journal of Education and Human Development, 6(4):17-29.

Vicari, R. M., Ribeiro, A., da Silva, J. M. C., Santos, E. R., Primo, T., and Bez, M. (2010). Brazilian proposal for agent-based learning objects metadata standard-obaa. In Metadata and Semantic Research, pages 300-311. Springer.

Wiley, D. A. (2000). Learning object design and sequencing theory. $\mathrm{PhD}$ thesis, Brigham Young University. 